\title{
A Note on Restricted Online Ramsey Numbers of Matchings
}

\author{
Vojtěch Dvořák* \\ Department of Pure Mathematics and Mathematical Statistics \\ University of Cambridge \\ Cambridge, United Kingdom \\ vd273@cam.ac.uk
}

Submitted: Nov 17, 2020; Accepted: Jun 7, 2021; Published: Jul 16, 2021

(C) The author. Released under the CC BY-ND license (International 4.0).

\begin{abstract}
Consider the following game between Builder and Painter. We take some families of graphs $\mathcal{G}_{1}, \ldots, \mathcal{G}_{t}$ and an integer $n$ such that $n \geqslant R\left(\mathcal{G}_{1}, \ldots, \mathcal{G}_{t}\right)$. In each turn, Builder picks an edge of initially uncoloured $K_{n}$ and Painter colours that edge with some colour $i \in\{1, \ldots, t\}$ of her choice. The game ends when a graph $G_{i}$ in colour $i$ for some $G_{i} \in \mathcal{G}_{i}$ and some $i$ is created. The restricted online Ramsey number $\tilde{R}\left(\mathcal{G}_{1}, \ldots, \mathcal{G}_{t} ; n\right)$ is the minimum number of turns that Builder needs to guarantee the game to end.

In a recent paper, Briggs and Cox studied the restricted online Ramsey numbers of matchings and determined a general upper bound for them. They proved that for $n=3 r-1=R_{2}\left(r K_{2}\right)$ we have $\widetilde{R}_{2}\left(r K_{2} ; n\right) \leqslant n-1$ and asked whether this was tight. In this short note, we provide a general lower bound for these Ramsey numbers. As a corollary, we answer this question of Briggs and Cox, and confirm that for $n=3 r-1$ we have $\tilde{R}_{2}\left(r K_{2} ; n\right)=n-1$. We also show that for $n^{\prime}=4 r-2=R_{3}\left(r K_{2}\right)$ we have $\tilde{R}_{3}\left(r K_{2} ; n^{\prime}\right)=5 r-4$.
\end{abstract}

Mathematics Subject Classifications: 05C57

\section{Introduction}

For families of graphs $\mathcal{G}_{1}, \ldots, \mathcal{G}_{t}$, the Ramsey number $R\left(\mathcal{G}_{1}, \ldots, \mathcal{G}_{t}\right)$ is the smallest integer $n$ such that any colouring of edges of $K_{n}$ with colours $1, \ldots, t$ contains a graph $G_{i}$ in colour $i$ for some $G_{i} \in \mathcal{G}_{i}$ and some $i \in\{1, \ldots, t\}$. When each family $\mathcal{G}_{i}$ contains a single graph $G_{i}$, we instead use the notation $R\left(G_{1}, \ldots, G_{t}\right)$ for the corresponding Ramsey number. When moreover we have $G_{1}=\cdots=G_{t}$, we use the notation $R_{t}\left(G_{1}\right)$ for $R\left(G_{1}, \ldots, G_{t}\right)$.

\footnotetext{
*The author was suported by the UK Engineering and Physical Sciences Research Council.
} 
The Ramsey numbers of graphs have been studied extensively, see for instance a survey of Conlon, Fox and Sudakov [6].

Many variants of the Ramsey numbers have been considered. One of them are the so-called online Ramsey numbers, introduced by Beck [1] and later independently by Kurek and Ruciński [12]. For families of graphs $\mathcal{G}_{1}, \ldots, \mathcal{G}_{t}$, the online Ramsey number $\tilde{R}\left(\mathcal{G}_{1}, \ldots, \mathcal{G}_{t}\right)$ is the smallest integer $k$ for which Builder can always guarantee a win within the first $k$ moves of the following game between Builder and Painter. Initially, we are given an infinite set of vertices, with every two vertices connected by an uncoloured edge. In each turn, Builder picks an edge between some two vertices in our set and Painter chooses any colour out of $1, \ldots, t$ and colours the edge with this colour. Builder wins once there is a graph $G_{i}$ in colour $i$ for some $G_{i} \in \mathcal{G}_{i}$ and some $i \in\{1, \ldots, t\}$. For various results about online Ramsey numbers, see $[4,5,8,9,11,13,14]$.

In 2008, Prałat [15] also introduced the restricted online Ramsey numbers (under different name, the name restricted online Ramsey numbers was first used for these by Conlon, Fox, Grinshpun and He [5]). These correspond to the same game as the online Ramsey numbers, but this game is now instead played on a finite board. To define this formally, for families of graphs $\mathcal{G}_{1}, \ldots, \mathcal{G}_{t}$ and an integer $n$ such that $n \geqslant R\left(\mathcal{G}_{1}, \ldots, \mathcal{G}_{t}\right)$, the restricted online Ramsey number $\tilde{R}\left(\mathcal{G}_{1}, \ldots, \mathcal{G}_{t} ; n\right)$ is the smallest integer $l$ for which Builder can always guarantee a win within the first $l$ moves of the following game between Builder and Painter. In each turn, Builder picks an edge of initially uncoloured $K_{n}$ and Painter chooses any colour out of $1, \ldots, t$ to colour this edge. Builder wins once there appears a graph $G_{i}$ in colour $i$ for some $G_{i} \in \mathcal{G}_{i}$ and some $i \in\{1, \ldots, t\}$. We note that the definitions of $\tilde{R}\left(\mathcal{G}_{1}, \ldots, \mathcal{G}_{t} ; n\right)$ differ slightly between the previous papers on this topic $[2,5,7,10,15]$, but it is easy to see that all are equivalent.

Analogously to the usual Ramsey numbers, when each family $\mathcal{G}_{i}$ contains a single graph $G_{i}$, we use the notation $\tilde{R}\left(G_{1}, \ldots, G_{t} ; n\right)$ for the corresponding restricted online Ramsey number. And when we further have $G_{1}=\cdots=G_{t}$, we use the notation $\tilde{R}_{t}\left(G_{1} ; n\right)$ for $\tilde{R}\left(G_{1}, \ldots, G_{t} ; n\right)$.

Briggs and Cox [2] studied the restricted online Ramsey numbers of matchings and trees. Before stating their results, recall the following well-known result of Cockayne and Lorimer [3] about the Ramsey numbers of matchings.

Theorem 1. For any $t \geqslant 2$ and positive integers $r_{1}, \ldots, r_{t}$, we have

$$
R\left(r_{1} K_{2}, \ldots, r_{t} K_{2}\right)=\max _{i} r_{i}+1+\sum_{i=1}^{t}\left(r_{i}-1\right) .
$$

Hence in particular, $R_{t}\left(r K_{2}\right)=r+1+t(r-1)$.

When $r$ is fixed, we will denote by $n_{t}$ for $t \geqslant 2$ the number $R_{t}\left(r K_{2}\right)=r+1+t(r-1)$. So in particular we have $n_{2}=3 r-1, n_{3}=4 r-2$ and $n_{4}=5 r-3$. Now we are ready to state the result of Briggs and Cox [2].

Theorem 2. Fix $t \geqslant 2$ and positive integers $r_{1}, \ldots, r_{t}$. If $n \geqslant R\left(r_{1} K_{2}, \ldots, r_{t} K_{2}\right)$, then 


$$
\tilde{R}\left(r_{1} K_{2}, \ldots, r_{t} K_{2} ; n\right) \leqslant \frac{2 t-1+(t-3) \log _{2}(t-2)}{t+1} n
$$

with the convention that $\log _{2} 0=0$.

Moreover, if we fix $r \geqslant 1$, then $\tilde{R}_{2}\left(r K_{2} ; n_{2}\right) \leqslant 3 r-2=n_{2}-1, \tilde{R}_{3}\left(r K_{2} ; n_{3}\right) \leqslant 5 r-4$ and $\tilde{R}_{4}\left(r K_{2} ; n_{4}\right) \leqslant 7 r-5$.

They ask whether we have $\tilde{R}_{2}\left(r K_{2} ; n_{2}\right)=n_{2}-1$. The aim of this short note is to verify that this indeed holds. We also show that the bound $\tilde{R}_{3}\left(r K_{2} ; n_{3}\right) \leqslant 5 r-4$ is tight and that the bound $\tilde{R}_{4}\left(r K_{2} ; n_{4}\right) \leqslant 7 r-5$ is tight except possibly for the exact value of the additive constant.

By describing a suitable strategy of Painter, we prove the following more general lower bound and a corollary about restricted online Ramsey numbers of matchings with few colours. This in particular answers the question of Briggs and Cox [2].

Theorem 3. Fix $t \geqslant 2$ and positive integers $r_{1}, \ldots, r_{t}$. If $n \geqslant R\left(r_{1} K_{2}, \ldots, r_{t} K_{2}\right)$, then $\tilde{R}\left(r_{1} K_{2}, \ldots, r_{t} K_{2} ; n\right) \geqslant 3\left(\sum_{i=1}^{t} r_{i}-t+1\right)-n$.

Hence if we fix $r \geqslant 1$, then $\tilde{R}_{2}\left(r K_{2} ; n_{2}\right)=3 r-2=n_{2}-1, \tilde{R}_{3}\left(r K_{2} ; n_{3}\right)=5 r-4$ and $\tilde{R}_{4}\left(r K_{2} ; n_{4}\right) \in\{7 r-6,7 r-5\}$.

It remains unclear whether for $t$ and $r$ large, the magnitude of $\tilde{R}_{t}\left(r K_{2} ; n_{t}\right)$ is closer to the upper bound from Theorem 2 or to the lower bound from Theorem 3.

\section{Proof of Theorem 3}

Consider the game played with $t$ colours on the edges of an initially uncoloured $K_{n}$. To prove Theorem 3, we will describe a strategy of Painter that ensures that after $T=$ $3\left(\sum_{i=1}^{t} r_{i}-t+1\right)-n-1$ moves (where by a move we mean Builder choosing some still uncoloured edge and Painter colouring it), there is no $r_{i} K_{2}$ of colour $i$ for $i=1, \ldots, t$.

While taking her turns (and to help her with her colouring decisions), Painter will moreover assign the following states to the coloured edges of $K_{n}$ and to all the vertices of $K_{n}$. Coloured edges are either free, or rooted. Every rooted edge is characterized by its root, which is one of its endpoints. Painter will assign (and update) the states of the coloured edges according to the strategy described below.

Vertices are of three types, characterized in the following way.

- If a vertex $v$ is a root of at least one coloured edge, it is of type I.

- If a vertex $v$ is not of type $\mathrm{I}$, but there is at least one free edge with endpoint $v$, it is of type II.

- If a vertex $v$ is neither of type I nor of type II, it is of type III.

In particular, note that initially all the vertices are of type III, since no edges are coloured at the start of the game. 
For $0 \leqslant j \leqslant\left(\begin{array}{l}n \\ 2\end{array}\right)$ and $i=1, \ldots, t$, let $A_{j}(i)$ be a number of type I vertices that are roots to at least one edge of colour $i$ after $j$ moves and let $B_{j}(i)$ be a number of free edges of colour $i$ after $j$ moves. Let $A_{j}=\sum_{i=1}^{t} A_{j}(i)$ and $B_{j}=\sum_{i=1}^{t} B_{j}(i)$.

Assume Builder chooses the edge $a b$ in $(k+1)$ st turn of his (where $0 \leqslant k \leqslant\left(\begin{array}{l}n \\ 2\end{array}\right)-1$ ). Without loss of generality (as we could otherwise switch $a$ and $b$ ), we can assume that if $b$ is of type I, then $a$ is also of type I; and if $b$ is of type II, then $a$ is of type I or of type II. Painter chooses the colour of an edge and updates the states of the coloured edges as follows.

(i) If $a$ is a vertex of type I, we declare the edge $a b$ to be rooted at $a$. By definition, there exists at least one other edge rooted at $a$, of some colour $c_{1}$ (if there are more edges rooted at $a$, pick one arbitrarily). We colour $a b$ by colour $c_{1}$.

(ii) If $a$ is a vertex of type II, there exists by definition a free edge $a c$ for some $c$, of some colour $c_{2}$ (if there are more free edges with endpoint $a$, pick one arbitrarily). We declare both edges $a b, a c$ to be rooted at $a$ and colour $a b$ in $c_{2}$.

(iii) If $a$ is a vertex of type III, then the edge $a b$ is declared to be free. It is coloured in any colour $c_{3}$ such that $A_{k}\left(c_{3}\right)+B_{k}\left(c_{3}\right) \leqslant r_{c_{3}}-2$ if at least one such colour exists, and if not in an arbitrary colour.

The next two observations are straightforward.

Observation 4. The number of vertices of type III:

- stays the same during move (i)

- increases by 1 or stays the same during move (ii)

- decreases by 2 during move (iii)

Observation 5. If move $j$ was $(i)$ or $(i i)$, we have $A_{j}(i)+B_{j}(i)=A_{j-1}(i)+B_{j-1}(i)$ for $i=1, \ldots, t$. If move $j$ was (iii) and Painter used colour $c$, we have $A_{j}(c)+B_{j}(c)=$ $A_{j-1}(c)+B_{j-1}(c)+1$ and for any $c^{\prime} \neq c$ we have $A_{j}\left(c^{\prime}\right)+B_{j}\left(c^{\prime}\right)=A_{j-1}\left(c^{\prime}\right)+B_{j-1}\left(c^{\prime}\right)$.

Using Observation 4 and Observation 5, we prove the key lemma.

Lemma 6. We have $A_{T}+B_{T} \leqslant \sum_{i=1}^{t} r_{i}-t$.

Proof. Let $C_{2}$ be the number of moves (ii) up to time $T$, and let $C_{3}$ be the number of moves (iii) up to time $T$. At time $T$, by Observation 4 we have at most $n+C_{2}-2 C_{3}$ vertices of type III. That implies $n+C_{2}-2 C_{3} \geqslant 0$. Since we further have $C_{2}+C_{3} \leqslant T$, we must have $C_{3} \leqslant \frac{n+T}{3}$.

Now by Observation $5, A_{T}+B_{T} \leqslant C_{3} \leqslant \frac{n+T}{3}=\sum_{i=1}^{t} r_{i}-t+\frac{2}{3}$, and since $A_{T}+B_{T}$ is an integer, we have $A_{T}+B_{T} \leqslant \sum_{i=1}^{t} r_{i}-t$ as required. 
Continuing the proof of Theorem 3 , we are now ready to show that after $T$ moves, there is no $r_{i} K_{2}$ of colour $i$ for $i=1, \ldots, t$.

Note that the existence of $r_{m} K_{2}$ of colour $m$ would in particular imply that $A_{T}(m)+$ $B_{T}(m) \geqslant r_{m}$. Because of the strategy of Painter and Observation 5, that would imply that $A_{T}(i)+B_{T}(i) \geqslant r_{i}-1$ for $i=1, \ldots, t$. Hence we would have $A_{T}+B_{T} \geqslant\left(r_{1}-1\right)+$ $\cdots+r_{m}+\cdots+\left(r_{t}-1\right)=\sum_{i=1}^{t} r_{i}-t+1$, contradicting Lemma 6 . Thus the proof of Theorem 3 is finished.

\section{Acknowledgements}

The author would like to thank his PhD supervisor Professor Béla Bollobás and anonymous referees for their helpful comments.

\section{References}

[1] J. Beck, Achievement games and the probabilistic method, Combinatorics, Paul Erdös is Eighty 1 (1993), 51-78.

[2] J. Briggs, C. Cox, Restricted online Ramsey numbers of matchings and trees, The Electronic Journal of Combinatorics 27(3) (2020), \#P3.49.

[3] E. Cockayne and P. J. Lorimer, The Ramsey number for stripes, Journal of the Australian Mathematical Society 19(2) (1975), 252-256.

[4] D. Conlon, Online Ramsey numbers, SIAM Journal of Discrete Mathematics 23 (2009), 1954-63.

[5] D. Conlon, J. Fox, A. Grinshpun, X. He, Online Ramsey numbers and the subgraph query problem, In Building Bridges II (2019), 159-164.

[6] D. Conlon, J. Fox, B. Sudakov, Recent developments in graph Ramsey theory, In A. Czumaj, A. Georgakopoulos, D. Král, V. Lozin, O. Pikhurko (Eds.), Surveys in Combinatorics (2015) 49-118.

[7] J. Cyman, T. Dzido, A note on on-line Ramsey numbers for quadrilaterals, Opuscula Mathematica 34 (2014), 463-468.

[8] J. Cyman, T. Dzido, J. Lapinskas, A. Lo, On-line Ramsey number of paths and cycles, The Electronic Journal of Combinatorics 22(1) (2015), \#P1.15.

[9] J. Dybizbański, T. Dzido, R. Zakrzewska, On-line Ramsey numbers for paths and short cycles, Discrete Applied Mathematics 282 (2020), 265-270.

[10] D. Gonzalez, X. He, H. Zheng, An upper bound for the restricted online Ramsey number, Discrete Mathematics 342(9) (2019), 2564-2569.

[11] J. Grytczuk, H. Kierstead, P. Prałat, On-line Ramsey Numbers for Paths and Stars, Discrete Mathematics and Theoretical Computer Science 10 (2008), 63-74.

[12] A. Kurek, A. Ruciński, Two variants of the size Ramsey number, Discuss. Math. Graph Theory 25 (2005), 141-149. 
[13] P. Prałat, A note on off-diagonal small on-line Ramsey numbers for paths, Ars Combinatoria 107 (2012), 295-306.

[14] P. Prałat, A note on small on-line Ramsey numbers for paths and their generalization, Australasian Journal of Combinatorics 40 (2008), 27-36.

[15] P. Prałat, $\overline{\mathcal{R}}(3,4)=17$, The Electronic Journal of Combinatorics 15 (2008), \#R67. 